\title{
EDITORIAL \\ Clipping of neurosurgical aneurysms: the dye is cast
}

\author{
Gregory J. Zipfel, MD
}

Departments of Neurological Surgery and Neurology, Washington University School of Medicine, St. Louis, Missouri

$\mathrm{I}$ $\mathrm{n}$ this issue Aaron Cohen-Gadol and colleagues report on a prospective study examining the utility of microscope-integrated intravenous fluorescein videoangiography (FL-VA) versus microscope-integrated intravenous indocyanine green videoangiography (ICG-VA) as intraoperative assessment tools to determine the safety and efficacy of surgical aneurysm obliteration. ${ }^{1}$ As the authors state, ICG-VA has become an invaluable tool in assessing the completeness of aneurysm obliteration and the patency of neighboring parent and branch arteries during aneurysm surgery, and in select cases can be used to supplant the need for intraoperative catheter angiography. ICG-VA, however, has several limitations. First, because it utilizes near-infrared light having wavelengths longer than visible light, the vascular images obtained with ICG-VA do not permit covisualization of fluorescent and nonfluorescent tissue through the operating microscope. This prevents real-time manipulation of vessels and brain tissue while the surgeon assesses ICG-VA results - a significant limitation, especially when assessing adequacy of aneurysm clipping in areas with narrow fields of view (e.g., anterior communicating artery and basilar apex regions) and for those surgeons who have adopted a retractorless surgical technique. I have personally found this to be a real limitation when using ICG-VA for assessing adequacy of aneurysm clipping. Second, due to an optical issue well explained in the article (chromatic aberration), ICG-VA images degrade at higher magnification and within deep corridors - a problem that I have also personally experienced on a number of occasions. To address these limitations, the authors first optimized the parameters of intravenous FL-VA, and second they prospectively assessed its utility compared with that of ICG-VA. They demonstrated that FL-VA provides very nice intraoperative assessment of vascular anatomy and adequacy of aneurysm clip placement. The clear advantage of this technique over ICG-VA is the ability to examine the vascular anatomy through the operating microscope (rather than on a nearby video screen that is the standard for ICG-VA). This permits real-time manipulation of the vasculature and surrounding brain tissue to achieve optimal visualization during fluorescent videoangiography. Previously, my colleagues and I compared the utility of ICG-VA versus intraoperative catheter angiography for aneurysm surgery and found a significant rate of discordance between the two modalities (14.3\%; defined as the need for aneurysm clip adjustment after ICG-VA), which suggested adequate aneurysm clipping, but intraoperative catheter angiography revealed residual aneurysm and/or vessel compromise. ${ }^{2}$ One of the primary drivers for this discordance was the presence of residual aneurysm or compromised vasculature that was obscured by the field of view provided by ICG-VA. It seems likely that at least some of these discordant cases could have been avoided if real-time manipulation of the vasculature during fluorescent videoangiography were possible. Another advantage of FL-VA that is well documented in the present paper is the improved visualization over ICG-VA in deep operative corridors. Here again, a limitation of ICG-VA - degraded image quality in deep operative corridors - may have contributed to our documented difficulty with using ICG-VA in the anterior communicating artery region. However, whether FL-VA will adequately address the issues we and others have encountered during surgery in the anterior communicating artery 
region (as well as similarly difficult-to-assess regions like the basilar apex) will require additional studies in which FL-VA is directly compared to the gold-standard intraoperative catheter angiography. Of course, these advantages of FL-VA are not without limitations, as the fluorescence associated with FL-VA lasts longer than ICG-VA (which makes repeat FL-VA more difficult) and the dura enhances more vividly with FL-VA than with ICG-VA (which in select cases may complicate assessment of aneurysm sac obliteration). Overall, the authors provide a very nice advancement and assessment to a previously reported fluorescent videoangiography technique that will likely find an important place in the armamentarium available to neurosurgeons to intraoperatively determine the adequacy and safety of aneurysm clip application. They are to be congratulated for this important contribution to the literature.

http://thejns.org/doi/abs/10.3171/2014.8.JNS14957

\section{References}

1. Lane B, Bohnstedt BN, Cohen-Gadol AA: A prospective comparative study of microscope-integrated intraoperative fluorescein and indocyanine videoangiography for clip ligation of complex cerebral aneurysms. J Neurosurg [epub ahead of print December 19, 2014. DOI: 10.3171/2014.10. JNS132766]

2. Washington CW, Zipfel GJ, Chicoine MR, Derdeyn CP, Rich KM, Moran CJ, et al: Comparing indocyanine green videoangiography to the gold standard of intraoperative digital subtraction angiography used in aneurysm surgery. $\mathbf{J}$ Neurosurg 118:420-427, 2013

\section{Response}

Aaron A. Cohen-Gadol, MD, MSc, ${ }^{1,2}$ Brandon Lane, MD, ${ }^{1}$ and Bradley N. Bohnstedt, MD ${ }^{3}$

${ }^{1}$ Goodman Campbell Brain and Spine, Department of Neurological Surgery, Indiana University; ${ }^{2}$ Indiana University Simon Cancer Center, Indianapolis, Indiana; and ${ }^{3}$ Department of Neurosurgery, University of Oklahoma Health Sciences Center, Oklahoma City, Oklahoma

We sincerely appreciate the astute summary of our work by Dr. Zipfel, who is one of the preeminent leaders in cerebrovascular neurosurgery. His editorial is a welcome and special addition to our work. He has encapsulated the germane results of our paper and has further expanded on potential future endeavors to compare FL-VA and intraoperative catheter angiography.

The introduction and evolution of minimally invasive exposures in cerebrovascular surgery has further tested the quality of fluorescent technologies. The use of supraorbital eyebrow incisions to treat anterior communicating aneurysms with clip ligation is one example of such evolution. ICG-VA unfortunately cannot be effective in these circumstances since inadequate exposure of the contrast agent to excitation light (due to limited operative space) in addition to the phenomenon of chromatic aberration renders ICG-VA ineffective in many instances.

Nonetheless, neither of these technologies is flawless, and we advocate discreet and complementary application of these fluorescent videoangiography techniques. 